PROCEEDINGS OF THE

AMERICAN MATHEMATICAL SOCIETY

Volume 139, Number 10, October 2011, Pages 3753-3762

S 0002-9939(2011)10814-1

Article electronically published on February 28, 2011

\title{
LAW OF LARGE NUMBERS UNDER THE NONLINEAR EXPECTATION
}

\author{
B. YANG AND H. XIAO
}

(Communicated by Edward C. Waymire)

\begin{abstract}
In this paper, we propose a class of nonlinear expectations induced by backward stochastic differential equations and reflected backward stochastic differential equations and prove the law of large numbers under the nonlinear expectation.
\end{abstract}

\section{INTRODUCTION}

The nonlinear backward stochastic differential equation (BSDE, for short) was firstly introduced by Pardoux and Peng [8. The theory of BSDE has wide applications to many fields such as financial mathematics, stochastic control, and partial differential equations (see [5]). In [4], El Karoui et al. generalized the results to BSDE's with reflection and introduced the notion of reflected backward stochastic differential equations (RBSDEs, for short). Their work linked a mixed optimal stopping-optimal stochastic control problem and a parabolic partial differential equation. Cvitanic and Karatzas $[3$ generalized the above results to the case of two reflecting barrier processes and establish the connection between RBSDE and certain stochastic games of stopping (Dynkin games).

One of the applications of BSDE, a nonlinear expectation, called the g-expectation, was introduced via BSDE by Peng 9. The g-expectation is monotone and constant-preserving, but not additive. In the setting of the g-expectation, many results of classic probability theory can be extended to some more general cases (see [1, 2], 6], 7], 9] and the references therein). In many cases, however, there are no constant-preserving expectations. For example, in the Black-Scholes market, the pricing process generated by the Black-Scholes formula, which can be viewed as a special nonlinear expectation, is not constant-preserving. For both BSDE and RBSDE, we will introduce new nonlinear expectations which are not constantpreserving and study the weak law of large numbers (LLN, for short) under our nonlinear expectations.

Received by the editors May 20, 2010 and, in revised form, September 4, 2010.

2010 Mathematics Subject Classification. Primary 60F05, 60G50; Secondary 60F99.

Key words and phrases. Law of large numbers, nonlinear expectation, backward stochastic differential equation, reflected backward stochastic differential equation.

The second author is the corresponding author and acknowledges the support of the National Nature Science Foundation of China (11001156, 11071144, 11026125), the Nature Science Foundation of Shandong Province (ZR2009AQ017), and the Independent Innovation Foundation of Shandong University (IIFSDU), China. 
The rest of the paper is organized as follows. In Section 2, the LLN problem under the nonlinear expectation is formulated. Section 3 introduces some preliminaries and lemmas. In Section 4, the LLN under the nonlinear expectation induced by BSDE or RBSDE respectively is established.

\section{Formulation of LLN PRoblem}

Let $\left(\Omega, \mathcal{F}, \mathcal{F}_{t}, \mathcal{P}\right)$ be a filtered complete probability space on which a standard Brownian motion $\left\{B_{t} ; 0 \leq t \leq T\right\}$ is defined, where $\left(\mathcal{F}_{t}\right)_{0 \leq t \leq T}$ is the natural filtration generated by $\left(B_{t}\right)$ and augmented with $\mathcal{P}$-null sets of $\mathcal{F}$. Let $\xi$ be an $\mathcal{F}_{T}$-measurable 1-dimensional random variable. Let us now introduce some spaces which will be used frequently in the sequel:

$$
\begin{aligned}
L^{2} & =\left\{\xi ; \xi \text { is an } \mathcal{F}_{T}-\text { measurable random variable s.t. } E\left(\xi^{2}\right)<\infty\right\} ; \\
H^{2} & =\left\{\varphi_{t} ;\left\{\varphi_{t}, 0 \leq t \leq T\right\} \text { is a predictable process s.t. } E \int_{0}^{T}\left|\varphi_{t}\right|^{2}<+\infty\right\} \\
\mathcal{S}^{2} & =\left\{\varphi_{t} ;\left\{\varphi_{t}, 0 \leq t \leq T\right\} \text { is a predictable process s.t. } E\left(\sup _{0 \leq t \leq T}\left|\varphi_{t}\right|^{2}\right)<+\infty\right\} .
\end{aligned}
$$

Pardoux and Peng [8] introduced the following nonlinear BSDE:

$$
y_{t}=\xi+\int_{t}^{T} g\left(s, y_{s}, z_{s}\right) d s-\int_{t}^{T} z_{s} d B_{s}, 0 \leq t \leq T
$$

where the function $g(t, y, z):[0, T] \times R \times R \rightarrow R$ is $\mathcal{F}_{t}$-progressively measurable for each $(y, z) \in R \times R$. It is by now well known that equation (2.1) has a unique adapted solution

$$
\left(y_{t}^{\xi}, z_{t}^{\xi}\right)_{t \in[0, T]} \in \mathcal{S}^{2} \times H^{2}
$$

if $\xi$ and $g$ satisfy the following assumptions:

$\left(\mathrm{A}_{1}\right) g(\cdot, y, z) \in L^{2}\left(\mathcal{F}_{T}\right),(y, z) \in R^{2}, \xi \in L^{2}\left(\mathcal{F}_{T}\right)$;

$\left(\mathrm{A}_{2}\right)\left|g\left(t, y_{1}, z_{1}\right)-g\left(t, y_{2}, z_{2}\right)\right| \leq K\left(\left|y_{1}-y_{2}\right|+\left|z_{1}-z_{2}\right|\right), t \in R, y_{1}, y_{2}, z_{1}, z_{2} \in R$.

We denote by $\left\{\left(y_{t}^{\xi}, z_{t}^{\xi}\right), t \in[0, T]\right\}$ the unique solution of BSDE (2.1). In addition, if $g$ satisfies the assumption

$\left(A_{3}\right) g(t, y, 0) \equiv 0,(t, y) \in[0, T] \times R$,

$y_{0}^{\xi}$ is called the $g$-expectation of $\xi$ in $[9$. The nonlinear $g$-expectation remains constant-preserving and monotone. The notion of $g$-expectation can be considered as a nonlinear extension of the well-known Girsanov transformations.

We now introduce the following assumption, which plays a key role in introducing the nonlinear expectation not preserving constants:

$$
\left(A_{3}^{\prime}\right) g(t, 0,0) \equiv 0, t \in[0, T] .
$$

Remark 2.1. An explanation of $\left(A_{3}^{\prime}\right)$ in the financial market is that a contingent claim in the future is zero. Then, under the pricing mechanism driven by $g(\cdot, \cdot, \cdot)$, the pricing process of the contingent claim is also zero.

We denote the solution $y_{t}^{\xi}$ of $\operatorname{BSDE}(2.1)$ by $E_{t}^{g}(\xi)$ and $y_{0}^{\xi}$ by $E^{g}(\xi)$ when $g$ satisfies the conditions $\left(A_{1}\right),\left(A_{2}\right)$ and $\left(A_{3}^{\prime}\right)$.

A solution of RBSDE (see El Karoui et al. [4]) is a triple $\left\{\left(Y_{t}, Z_{t}, K_{t}\right), 0 \leq t \leq T\right\}$ of $\mathcal{F}_{t}$-progressively measurable processes taking values in $R \times R \times R_{+}$, respectively, 
and satisfying:

$$
\left\{\begin{array}{l}
\text { (i) } Z \in H_{2}, E \int_{0}^{T}\left|Z_{t}\right|^{2}<+\infty \\
\text { (ii) } Y \in \mathcal{S}_{2} \text { and } K_{T} \in L^{2} \\
\text { (iii) } Y_{t}=\xi+\int_{t}^{T} g\left(s, Y_{s}, Z_{s}\right) d s+K_{T}-K_{t}-\int_{t}^{T} Z_{s} d B_{s}, 0 \leq t \leq T \\
\text { (iv) } Y_{t} \geq S_{t}, 0 \leq t \leq T \\
\text { (v) } K_{t} \text { is continuous and increasing, } K_{0}=0 \text { and } \int_{0}^{T}\left(Y_{t}-S_{t}\right) d K_{t}=0
\end{array}\right.
$$

where $S_{t}, 0 \leq t \leq T$, is a continuous progressively measurable process satisfying $E\left(\sup _{0 \leq t \leq T}\left|S_{t}\right|^{2}\right)<+\infty$. There exists a unique solution $\left(Y_{t}^{\xi}, Z_{t}^{\xi}, K_{t}^{\xi}\right)$ to the $\operatorname{RBSDE}(2.2)$, and we denote the solution $Y_{t}^{\xi}$ of RBSDE by $\mathcal{E}_{t}^{g}(\xi)$ and $Y_{0}^{\xi}$ by $\mathcal{E}^{g}(\xi)$ if $g$ satisfies $\left(A_{1}\right),\left(A_{2}\right)$ and $\left(A_{3}^{\prime}\right)$ (see [4]).

Hereinafter, we always assume that $g$ satisfies $\left(A_{1}\right),\left(A_{2}\right)$ and $\left(A_{3}^{\prime}\right)$. We call $E^{g}(\xi)$ and $\mathcal{E}^{g}(\xi)$ the corresponding nonlinear expectation of $\xi$. It is clear that the property of constant preservation does not hold for $E^{g}(\cdot)$ and $\mathcal{E}^{g}(\cdot)$.

Let $\xi_{1}, \xi_{2}, \ldots$ be a sequence of independent identically distributed random variables with finite second moment. Then Kolomogorov's strong law of large numbers holds; i.e.,

$$
\lim _{n \rightarrow \infty}\left(\frac{\xi_{1}+\xi_{2}+\cdots+\xi_{n}}{n}\right)=E\left(\xi_{1}\right), \quad \mathcal{P}-\text { a.s. },
$$

which can be viewed as the behavior of the random walk in the physical world $\left(\Omega, \mathcal{F}, \mathcal{F}_{t}, \mathcal{P}\right)$. But, for a special financial market $\left(\Omega, \mathcal{F}, \mathcal{F}_{t}, E^{g}\right)$ or $\left(\Omega, \mathcal{F}, \mathcal{F}_{t}, \mathcal{E}^{g}\right)$, we are more concerned with the question of whether

$$
\lim _{n \rightarrow \infty} E^{g}\left(\frac{\xi_{1}+\xi_{2}+\cdots+\xi_{n}}{n}\right)=E^{g}\left(E\left(\xi_{1}\right)\right)
$$

or

$$
\lim _{n \rightarrow \infty} \mathcal{E}^{g}\left(\frac{\xi_{1}+\xi_{2}+\cdots+\xi_{n}}{n}\right)=\mathcal{E}^{g}\left(E\left(\xi_{1}\right)\right)
$$

holds.

In this paper, we prove that the nonlinear expectation of the random variable $\left(\frac{\xi_{1}+\xi_{2}+\cdots+\xi_{n}}{n}\right)$ converges to the nonlinear expectation of $E\left(\xi_{1}\right)$. We call it the law of large numbers under the nonlinear expectation.

\section{Some PRELIMINARIES AND LEMMAS}

Let $L^{p}\left(\mathcal{F}_{T}\right)$ be the space of $\mathcal{F}_{T}$-measurable random variables $\xi$ satisfying $E|\xi|^{P}=$ $\int_{\Omega}|\xi|^{p} d \mathcal{P}<\infty$. Let $\xi_{1}, \xi_{2}, \ldots$ be a sequence of independent identically distributed random variables with finite second moments and $S_{n}=\xi_{1}+\xi_{2}+\cdots+\xi_{n}, n=1,2, \cdots$.

We first introduce several lemmas.

Lemma 3.1 (see [10], p. 389). If there are positive numbers $b_{n}$ such that $b_{n} \uparrow \infty$ and $\sum \frac{V \xi_{n}}{b_{n}^{2}}<\infty$, then $\frac{S_{n}-E S_{n}}{b_{n}} \rightarrow 0, \mathcal{P}-$ a.s. In particular, if $\sum \frac{V \xi_{n}}{n^{2}}<\infty$, then $\frac{S_{n}-E S_{n}}{n} \rightarrow 0, \mathcal{P}-$ a.s. 
The following assumption has a very good interpretation in finance.

$\left(A_{4}\right)$ For each constant $K,\left\{E_{t}^{g}(K) ; t \in[0, T]\right\}\left(\left\{\mathcal{E}_{t}^{g}(K) ; t \in[0, T]\right\}\right)$ is a deterministic function in $t$.

Remark 3.2. In the financial market $\left(\Omega, \mathcal{F}, \mathcal{F}_{t}, E^{g}\right)$, for each constant $K,\left\{E_{t}^{g}(K)\right.$; $t \in[0, T]\}$ may be viewed as the pricing process of the future risk-free deterministic asset $K$. The assumption $\left(A_{4}\right)$ means that the pricing process of the future risk-free asset $K$ is a deterministic function in $t$ and that if $K \neq 0$, then $E_{t}^{g}(K) \neq 0$. A similar interpretation can be made for $\mathcal{E}_{t}^{g}(K)$.

We write $g_{0}(y)=g(y, 0)$ and $g_{1}(y, z)=g(y, z)-g_{0}(y)$. It is easy to show that $g_{0}(0)=0, g_{1}(y, 0)=0$, and both $g_{0}(y)$ and $g_{1}(y, z)$ satisfy the conditions $\left(A_{1}\right),\left(A_{2}\right)$ and $\left(A_{3}^{\prime}\right)$.

Lemma 3.3. If $g_{0}$ satisfies $\left(A_{4}\right)$, then $g$ also satisfies $\left(A_{4}\right)$ and $E_{t}^{g_{0}}(K)=E_{t}^{g}(K)$. Proof. For a given constant $K \in R, 0 \leq t \leq T$, we have

$$
\begin{aligned}
E_{t}^{g_{0}}(K) & =K+\int_{t}^{T} g_{0}\left(E_{s}^{g_{0}}(K)\right) d s-\int_{t}^{T} 0 d B_{s} \\
& =K+\int_{t}^{T}\left(g_{0}\left(E_{s}^{g_{0}}(K)\right)+g_{1}(0,0)\right) d s-\int_{t}^{T} 0 d B_{s} \\
& =K+\int_{t}^{T}\left(g_{0}\left(E_{s}^{g_{0}}(K)\right)+g_{1}\left(E_{s}^{g_{0}}(K), 0\right)\right) d s-\int_{t}^{T} 0 d B_{s} \\
& =K+\int_{t}^{T} g\left(E_{s}^{g_{0}}(K), 0\right) d s-\int_{t}^{T} 0 d B_{s} .
\end{aligned}
$$

By the uniqueness of the solution of BSDE (2.1), we obtain $E_{t}^{g_{0}}(K)=E_{t}^{g}(K)$, which is also a deterministic function.

\section{MAIN RESUltS}

Let $\xi_{1}, \xi_{2}, \cdots$ be a sequence of independent identically distributed random variables with finite second moments. Set $C=E\left(\xi_{1}\right)$ and $S_{n}=\xi_{1}+\xi_{2}+\cdots+\xi_{n}$, $n=1,2, \cdots$.

4.1. LLN under the nonlinear expectation induced by BSDE. We are in a position to prove that LLN holds under the nonlinear expectation induced by BSDE .

Theorem 4.1. If $g$ satisfies $\left(A_{1}\right),\left(A_{2}\right)$ and $\left(A_{3}^{\prime}\right), g_{0}(y)$ satisfies $\left(A_{4}\right)$, and there exists a random variable $\eta \in L^{2}\left(\mathcal{F}_{T}\right)$ such that $\left|\xi_{i}\right| \leq|\eta|, i=1,2, \cdots$, then $\lim _{n \rightarrow \infty} E^{g}\left(\frac{S_{n}}{n}\right)=E^{g}(C)$.

Proof. Set $\frac{S_{n}}{n}=C+\varepsilon_{n}$. By Lemma 3.1, $\lim _{n \rightarrow \infty} \frac{S_{n}}{n}=C, \mathcal{P}$-a.s., i.e., $\lim _{n \rightarrow \infty} \varepsilon_{n}=$ $0, \mathcal{P}$-a.s. For $0 \leq t \leq T$, we have

$$
\begin{aligned}
& y_{t}=C+\int_{t}^{T} g_{0}\left(y_{s}\right) d s-\int_{t}^{T} 0 d B_{s}, \\
& y_{t}^{n}=\varepsilon_{n}+\int_{t}^{T} g\left(y_{s}^{n}, z_{s}^{n}\right) d s-\int_{t}^{T} z_{s}^{n} d B_{s}, \\
& \overline{y_{t}^{n}}=\left(C+\varepsilon_{n}\right)+\int_{t}^{T} g\left(\overline{y_{s}^{n}}, \overline{z_{s}^{n}}\right) d s-\int_{t}^{T} \overline{z_{s}^{n}} d B_{s} ;
\end{aligned}
$$


that is,

$$
\begin{aligned}
d y_{t} & =-g_{0}\left(y_{t}\right) d t, & & y_{T}=C, \\
d y_{t}^{n} & =-g\left(y_{t}^{n}, z_{t}^{n}\right) d t+z_{t}^{n} d B_{t}, & & y_{T}^{n}=\varepsilon_{n}, \\
d \overline{y_{t}^{n}} & =-g\left(\overline{y_{t}^{n}}, \overline{z_{t}^{n}}\right) d t+\overline{z_{t}^{n}} d B_{t}, & & \overline{y_{T}^{n}}=C+\varepsilon_{n} .
\end{aligned}
$$

From (4.1), we obtain

$$
d\left(\overline{y_{t}^{n}}-y_{t}\right)=-\left(g\left(\overline{y_{t}^{n}}, \overline{z_{t}^{n}}\right)-g_{0}\left(y_{t}\right)\right) d t+\overline{z_{t}^{n}} d B_{t}, 0 \leq t \leq T .
$$

Denote $\widetilde{y_{t}^{n}}=\overline{y_{t}^{n}}-y_{t}$ and $\widetilde{z_{t}^{n}}=\overline{z_{t}^{n}}$. Applying Itô's formula to $e^{\beta t}\left(\widetilde{y_{t}^{n}}\right)^{2}$, we have

$$
\left\{\begin{aligned}
d\left(e^{\beta t}\left(\widetilde{y_{t}^{n}}\right)^{2}\right) & =e^{\beta t}\left[\beta\left(\widetilde{y_{t}^{n}}\right)^{2} d t-\widetilde{y_{t}^{n}}\left(a_{t} \widetilde{y_{t}^{n}}+b_{t} \widetilde{z_{t}^{n}}\right) d t+2 \widetilde{y_{t}^{n}} \widetilde{z_{t}^{n}} d B_{t}+{\widetilde{z_{t}^{n}}}^{2} d t\right], \\
e^{\beta T} \widetilde{y_{T}^{n}} & =\varepsilon_{n}^{2} e^{\beta T},
\end{aligned}\right.
$$

where

$$
\begin{gathered}
a_{t}= \begin{cases}\frac{\left(g\left(\overline{y_{t}^{n}}, \overline{z_{t}^{n}}\right)-g\left(y_{t}^{n}, \overline{z_{t}^{n}}\right)\right)}{\overline{y_{t}^{n}}-y_{t}}, & \text { if } \overline{y_{t}^{n}} \neq y_{t}, \\
0, & \text { else, }\end{cases} \\
b_{t}= \begin{cases}\frac{\left(g\left(y_{t}^{n}, \overline{z_{t}^{n}}\right)-g_{0}\left(y_{t}\right)\right)}{\bar{z}_{t}^{n}}, & \text { if } \bar{z}_{t}^{n} \neq 0, \\
0, & \text { else. }\end{cases}
\end{gathered}
$$

Integrating the first equality in (4.2) from $t$ to $T$, we arrive at

$$
\begin{aligned}
& \varepsilon_{n}^{2} e^{\beta T}-\left(e^{\beta t}\left(\widetilde{y_{t}^{n}}\right)^{2}\right) \\
= & \int_{t}^{T} e^{\beta s}\left(\widetilde{\beta y_{s}^{n}}{\widetilde{z_{s}^{n}}}^{2}-a_{s}{\widetilde{y_{s}^{n}}}^{2}-b_{s} \widetilde{y_{s}^{n}} \widetilde{z_{t}^{n}}\right) d s+2 \int_{t}^{T} e^{\beta s} \widetilde{y_{s}^{n}} \widetilde{z^{n}} d B_{s} .
\end{aligned}
$$

Then we get

$$
\begin{aligned}
& E\left(\varepsilon_{n}^{2} e^{\beta T} \mid \mathcal{F}_{t}\right)+E\left(\int_{t}^{T}\left(a_{s}{\widetilde{y_{s}^{n}}}^{2}+b_{s} \widetilde{y_{s}^{n}} \widetilde{z_{s}^{n}}\right) e^{\beta s} d s \mid \mathcal{F}_{t}\right) \\
= & \left(\widetilde{y_{t}^{n}}\right)^{2} e^{\beta t}+E\left(\int_{t}^{T}\left(\widetilde{\beta y_{s}^{n}}{ }^{2}+{\widetilde{z_{s}^{n}}}^{2}\right) e^{\beta s} d s \mid \mathcal{F}_{t}\right) .
\end{aligned}
$$

In terms of (4.4), it follows that

$$
\begin{aligned}
& {\widetilde{y_{t}^{n}}}^{2}+E\left(\int_{t}^{T} e^{\beta(s-t)}\left(\beta{\widetilde{y_{s}^{n}}}^{2}+{\widetilde{z_{s}^{n}}}^{2}\right) d s \mid \mathcal{F}_{t}\right) \\
= & E\left(\varepsilon_{n}{ }^{2} e^{\beta(T-t)} \mid \mathcal{F}_{t}\right)+E\left(\int_{t}^{T}\left(a_{s}{\widetilde{y_{s}^{n}}}^{2}+b_{s} \widetilde{y_{s}^{n}} \widetilde{z_{s}^{n}}\right) e^{\beta(s-t)} d s \mid \mathcal{F}_{t}\right) .
\end{aligned}
$$

Because $g$ satisfies $\left(\mathrm{A}_{1}\right),\left(\mathrm{A}_{2}\right),\left(\mathrm{A}_{3}^{\prime}\right)$ and $\left(\mathrm{A}_{4}\right)$, there exists a constant $K>0$ such that

$$
\left|a_{t}\right| \leq K,\left|b_{t}\right| \leq K, 0 \leq t \leq T
$$


Therefore,

$$
\begin{aligned}
& {\widetilde{y_{t}^{n}}}^{2}+E\left(\int_{t}^{T} e^{\beta(s-t)}\left(\widetilde{\beta y_{s}^{n}}+\widetilde{z_{s}^{n}}\right) d s \mid \mathcal{F}_{t}\right) \\
& \leq E\left(\varepsilon_{n}^{2} e^{\beta(T-t)} \mid \mathcal{F}_{t}\right)+K E\left(\int_{t}^{T} \widetilde{y_{s}^{n}} e^{\beta(s-t)} d s \mid \mathcal{F}_{t}\right) \\
& \quad+K E\left(\int_{t}^{T} e^{\beta(s-t)}\left|\widetilde{y_{s}^{n}} \widetilde{z_{s}^{n}}\right| d s \mid \mathcal{F}_{t}\right) .
\end{aligned}
$$

From

$$
\left|2 K \widetilde{y_{s}^{n}} \widetilde{z}_{s}^{n}\right| \leq \frac{\beta}{2}{\widetilde{y_{s}^{n}}}^{2}+\frac{2 K^{2}}{\beta}{\widetilde{z_{s}^{n}}}^{2}
$$

we have

$$
\begin{aligned}
& \left(\widetilde{y_{t}^{n}}\right)^{2}+E\left(\int_{t}^{T} e^{\beta(s-t)}\left(\beta{\widetilde{y_{s}^{n}}}^{2}+{\widetilde{z_{s}^{n}}}^{2}\right) d s \mid \mathcal{F}_{t}\right) \\
\leq & E\left(\varepsilon_{n}^{2} e^{\beta(T-t)} \mid \mathcal{F}_{t}\right) \\
& +\left(K+\frac{\beta}{4}\right) E\left(\int_{t}^{T} e^{\beta(s-t)}{\widetilde{y_{s}^{n}}}^{2} d s\left|\mathcal{F}_{t}+\frac{K^{2}}{\beta} e^{\beta(s-t)}{\widetilde{z_{s}^{n}}}^{2} d s\right| \mathcal{F}_{t}\right) .
\end{aligned}
$$

For a sufficiently large positive number $\beta$, it follows that there exist two positive numbers $K_{1}$ and $K_{2}$ such that

$$
\begin{aligned}
& \left(\widetilde{y_{t}^{n}}\right)^{2}+K_{1} E\left(\int_{t}^{T} e^{(s-t)} \beta \widetilde{y_{s}^{n}}{ }^{2} d s \mid \mathcal{F}_{t}\right)+K_{2} E\left(\int_{t}^{T} e^{(s-t)}{\widetilde{z_{s}^{n}}}^{2} d s \mid \mathcal{F}_{t}\right) \\
\leq & E\left(\varepsilon_{n}{ }^{2} e^{\beta(T-t)} \mid \mathcal{F}_{t}\right) .
\end{aligned}
$$

Letting $t=0$ in (4.7), we conclude that

$$
\left(\widetilde{y_{t}^{n}}\right)^{2}+K_{1} E \int_{t}^{T} e^{s} \beta{\widetilde{y_{s}^{n}}}^{2} d s+K_{2} E \int_{t}^{T} e^{s}{\widetilde{z_{s}^{n}}}^{2} d s \leq E\left(\varepsilon_{n}{ }^{2} e^{\beta T}\right)
$$

Because $\left|\xi_{i}\right| \leq|\eta|, i=1,2, \cdots, \eta \in L^{2}\left(\mathcal{F}_{T}\right)$, by the Lebesgue dominated convergence theorem, we have

$$
\lim _{n \rightarrow \infty} E\left(\varepsilon_{n}^{2}\right)=\lim _{n \rightarrow \infty} E\left(\frac{s_{n}}{n}-C\right)^{2}=0 .
$$

Therefore,

$$
\lim _{n \rightarrow \infty} \widetilde{y_{0}^{n}}=0
$$

i.e.,

$$
\lim _{n \rightarrow \infty} E^{g}\left(\frac{s_{n}}{n}\right)=E^{g}(C) .
$$

4.2. LLN under nonlinear expectation induced by RBSDE. Let $\left\{\mathcal{B}_{t}, 0 \leq\right.$ $t \leq T\}$ be a 1-dimensional standard Brownian motion defined on a probability space $\left(\Omega, \mathcal{F},\left\{\mathcal{F}_{t}\right\}, \mathcal{P}\right)$. We now prove the LLN under nonlinear expectation induced by RBSDE.

Theorem 4.2. If $g$ satisfies $\left(A_{1}\right),\left(A_{2}\right)$ and $\left(A_{3}^{\prime}\right), g_{0}(y)$ satisfies $\left(A_{4}\right)$, and there exists a random variable $\eta \in L^{2}\left(\mathcal{F}_{T}\right)$ such that $\left|\xi_{i}\right| \leq|\eta|, i=1,2, \cdots$, then

$$
\lim _{n \rightarrow \infty} \mathcal{E}^{g}\left(\frac{\xi_{1}+\xi_{2}+\cdots+\xi_{n}}{n}\right)=\mathcal{E}^{g}\left(E\left(\xi_{1}\right)\right) .
$$


Proof. As above, for $0 \leq t \leq T$, let

$$
\left\{\begin{array}{l}
y_{t}=C+\int_{t}^{T} g_{0}\left(y_{s}\right) d s+K_{T}-K_{t}-\int_{t}^{T} 0 d B_{s} \\
y_{t} \geq S_{t} \\
\int_{0}^{T}\left(y_{t}-S_{t}\right) d K_{t}=0
\end{array}\right.
$$

and

$$
\left\{\begin{array}{l}
\overline{y_{t}^{n}}=\left(C+\varepsilon_{n}\right)+\int_{t}^{T} g\left(\overline{y_{s}^{n}}, \overline{z_{s}^{n}}\right) d s+\overline{K_{T}}-\overline{K_{t}}-\int_{t}^{T} \overline{z_{s}^{n}} d B_{s} \\
\overline{y_{t}^{n}} \geq S_{t} \\
\int_{0}^{T}\left(\overline{y_{t}^{n}}-S_{t}\right) d K_{t}=0
\end{array}\right.
$$

From (4.9) and (4.10), we obtain

$$
\left\{\begin{aligned}
d\left(\overline{y_{t}^{n}}-y_{t}\right) & =-\left(g\left(\overline{y_{t}^{n}}, \overline{z_{t}^{n}}\right)-g_{0}\left(y_{t}\right)\right) d t+\overline{z_{t}^{n}} d B_{t}-d\left(\overline{K_{t}^{n}}-K_{t}\right), \\
\overline{y_{T}^{n}}-y_{T} & =\varepsilon_{n} .
\end{aligned}\right.
$$

Set $\widetilde{y_{t}^{n}}=\overline{y_{t}^{n}}-y_{t}, \widetilde{z_{t}^{n}}=\overline{z_{t}^{n}}, \widetilde{K_{t}^{n}}=\overline{K_{t}^{n}}-K_{t}$ and apply Itô's formula to $e^{\beta t}\left(\widetilde{y_{t}^{n}}\right)^{2}$. Then

$$
\left\{\begin{aligned}
d\left(e^{\beta t}\left(\widetilde{y_{t}^{n}}\right)^{2}\right)=e^{\beta t}\left[\beta\left(\widetilde{y_{t}^{n}}\right)^{2} d t-\widetilde{y_{t}^{n}}\left(a_{t} \widetilde{y_{t}^{n}}+b_{t} \widetilde{z_{t}^{n}}\right) d t\right. \\
\left.+2 \widetilde{y_{t}^{n}} \widetilde{z_{t}^{n}} d B_{t}+{\widetilde{z_{t}^{n}}}^{2} d t-2 \widetilde{y_{t}^{n}} d \widetilde{K_{t}^{n}}\right] \\
e^{\beta T}{\widetilde{y_{T}^{n}}}^{2}=\varepsilon_{n}{ }^{2} e^{\beta T},
\end{aligned}\right.
$$

where

$$
a_{t}= \begin{cases}\frac{\left(g\left(\overline{y_{t}^{n}}, \overline{z_{t}^{n}}\right)-g\left(y_{t}^{n}, \overline{z_{t}^{n}}\right)\right)}{\overline{y_{t}^{n}}-y_{t}}, & \text { if } \overline{y_{t}^{n}} \neq y_{t} \\ 0, & \text { else }\end{cases}
$$

and

$$
b_{t}= \begin{cases}\frac{\left(g\left(y_{t}^{n}, \overline{z_{t}^{n}}\right)-g_{0}\left(y_{t}\right)\right)}{\bar{z}_{t}^{n}}, & \text { if } \bar{z}_{t}^{n} \neq 0, \\ 0, & \text { else. }\end{cases}
$$

Integrating both sides of (4.11) from $t$ to $T$ yields

$$
\begin{aligned}
& \varepsilon_{n}{ }^{2} e^{\beta T}-\left(e^{\beta t}\left(\widetilde{y_{t}^{n}}\right)^{2}\right) \\
= & \int_{t}^{T} e^{\beta s}\left(\beta \widetilde{y_{s}^{n}}{ }^{2}+{\widetilde{z_{t}^{n}}}^{2}-a_{s}{\widetilde{y_{s}^{n}}}^{2}-b_{s} \widetilde{y_{s}^{n}} \widetilde{z_{t}^{n}}\right) d s \\
& +2 \int_{t}^{T} e^{\beta s} \widetilde{y_{s}^{n}} \widetilde{z^{n}} d s-2 \int_{t}^{T} e^{\beta s} \widetilde{y_{s}^{n}} \widetilde{d K_{t}^{n}} .
\end{aligned}
$$


Taking conditional expectation with respect to $\mathcal{F}_{t}$ in (4.12), we have

$$
\begin{aligned}
& {\widetilde{y_{t}^{n}}}^{2}+\mathcal{E}^{g}\left(\int_{t}^{T} e^{\beta(s-t)}\left(\widetilde{\beta y_{t}^{n}}{ }^{2}+{\widetilde{z_{s}^{n}}}^{2}\right) d s \mid \mathcal{F}_{t}\right) \\
= & \mathcal{E}^{g}\left(\varepsilon_{n}{ }^{2} e^{\beta(T-t)} \mid \mathcal{F}_{t}\right)+\mathcal{E}^{g}\left(\int_{t}^{T}\left(a_{s}{\widetilde{y_{s}^{n}}}^{2}+b_{s} \widetilde{y_{s}^{n}} \widetilde{z_{s}^{n}}\right) e^{\beta(s-t)} d s \mid \mathcal{F}_{t}\right) \\
& +2 \mathcal{E}^{g}\left[\int_{t}^{T} e^{\beta(s-t)} \widetilde{y_{s}^{n}} \widetilde{K_{s}^{n}} \mid \mathcal{F}_{t}\right] .
\end{aligned}
$$

Because $g$ satisfies $\left(\mathrm{A}_{1}\right)-\left(\mathrm{A}_{4}\right)$, there exists a constant $K$ such that

$$
\left|a_{t}\right| \leq K,\left|b_{t}\right| \leq K, 0 \leq t \leq T .
$$

Combining (4.13) with (4.14), we have

$$
\begin{aligned}
& {\widetilde{y_{t}^{n}}}^{2}+\mathcal{E}^{g}\left(\int_{t}^{T} e^{\beta(s-t)}\left(\widetilde{\beta y_{t}^{n}}+\widetilde{z_{s}^{n}}{ }^{2}\right) d s \mid \mathcal{F}_{t}\right) \\
\leq & \mathcal{E}^{g}\left(\varepsilon_{n}{ }^{2} e^{\beta(T-t)} \mid \mathcal{F}_{t}\right)+K \mathcal{E}^{g}\left(\int_{t}^{T}{\widetilde{y_{s}^{n}}}^{2} e^{\beta(s-t)} d s \mid \mathcal{F}_{t}\right) \\
& +K \mathcal{E}^{g}\left(\int_{t}^{T} e^{\beta(s-t)} \widetilde{y_{s}^{n}} \widetilde{z_{s}^{n}} d s \mid \mathcal{F}_{t}\right)+2 \mathcal{E}^{g}\left[\int_{t}^{T} e^{\beta(s-t)} \widetilde{y_{s}^{n}} d \widetilde{K_{s}^{n}} \mid \mathcal{F}_{t}\right]
\end{aligned}
$$

Since

$$
\left|2 K \widetilde{y_{s}^{n}} \widetilde{z}_{s}^{n}\right| \leq \frac{\beta}{2}{\widetilde{y_{s}^{n}}}^{2}+\frac{2 K^{2}}{\beta}{\widetilde{z_{s}^{n}}}^{2},
$$

we have

$$
\begin{aligned}
& \left(\widetilde{y_{t}^{n}}\right)^{2}+\mathcal{E}^{g}\left[\int_{t}^{T} e^{\beta(s-t)}\left(\beta{\widetilde{y_{t}^{n}}}^{2}+{\widetilde{z_{s}^{n}}}^{2}\right) d s \mid \mathcal{F}_{t}\right] \\
\leq & \mathcal{E}^{g}\left(\varepsilon_{n}^{2} e^{\beta(T-t)} \mid \mathcal{F}_{t}\right)+\left(K+\frac{\beta}{4}\right) \mathcal{E}^{g}\left(\int_{t}^{T} e^{\beta(s-t)}{\widetilde{y_{s}^{n}}}^{2} d s \mid \mathcal{F}_{t}\right) \\
& +\frac{K^{2}}{\beta} \mathcal{E}^{g}\left(\int_{t}^{T} e^{\beta(s-t)} \widetilde{z_{s}^{n}} d s \mid \mathcal{F}_{t}\right)+2 \mathcal{E}^{g}\left[\int_{t}^{T} e^{\beta(s-t)} \widetilde{y_{s}^{n}} d \widetilde{K_{s}^{n}} \mid \mathcal{F}_{t}\right]
\end{aligned}
$$

For a sufficiently large positive number $\beta$, it follows that there exist two positive numbers $K_{1}$ and $K_{2}$ such that

$$
\begin{aligned}
& \left(\widetilde{y_{t}^{n}}\right)^{2}+K_{1} \mathcal{E}^{g}\left(\int_{t}^{T} e^{(s-t)} \beta \widetilde{y_{s}^{n}} d s \mid \mathcal{F}_{t}\right)+K_{2} \mathcal{E}^{g}\left(\int_{t}^{T} e^{(s-t)} \widetilde{z_{s}^{n}} d s \mid \mathcal{F}_{t}\right) \\
\leq & \mathcal{E}^{g}\left(\varepsilon_{n}{ }^{2} e^{\beta(T-t)} \mid \mathcal{F}_{t}\right)+2 \mathcal{E}^{g}\left[\int_{t}^{T} e^{\beta(s-t)} \widetilde{y_{s}^{n}} d \widetilde{K_{s}^{n}} \mid \mathcal{F}_{t}\right] .
\end{aligned}
$$

Letting $t=0$ in (4.16), we conclude that

$$
\begin{aligned}
& \left(\widetilde{y_{0}^{n}}\right)^{2}+K_{1} \mathcal{E}^{g}\left(\int_{0}^{T} e^{s} \beta \widetilde{y_{s}^{n}} d s\right)+K_{2} \mathcal{E}^{g}\left(\int_{0}^{T} e^{s} \widetilde{z_{s}^{n}} d s\right) \\
\leq & e^{\beta T} \mathcal{E}^{g}\left(\varepsilon_{n}^{2}\right)+2 \mathcal{E}^{g} \int_{0}^{T} e^{\beta s} \widetilde{y_{s}^{n}} d \widetilde{K_{s}^{n}} .
\end{aligned}
$$


Since

$$
\begin{aligned}
\int_{0}^{T} \widetilde{y^{n}} d \widetilde{K_{t}^{n}}= & \int_{0}^{T}\left(\overline{y_{t}^{n}}-y_{t}\right) d K_{t}^{n}-\int_{0}^{T}\left(\overline{y_{t}^{n}}-y_{t}\right) d K_{t} \\
= & \int_{0}^{T}\left(\overline{y_{t}^{n}}-S_{t}^{n}\right) d K_{t}^{n}+\int_{0}^{T}\left(S_{t}^{n}-\overline{y_{t}^{n}}\right) d K_{t}^{n} \\
& -\int_{0}^{T}\left(\overline{y_{t}^{n}}-y_{t}\right) d K_{t}+\int_{0}^{T}\left(S_{t}^{n}-\overline{y_{t}^{n}}\right) d K_{t} \leq 0
\end{aligned}
$$

we have

$$
2 \mathcal{E}^{g}\left[\int_{0}^{T} e^{\beta(s-t)} \widetilde{y_{s}^{n}} d \widetilde{K_{s}^{n}} \mid \mathcal{F}_{t}\right] \leq 2 e^{\beta(-t)} \mathcal{E}^{g}\left[\int_{0}^{T} \widetilde{y_{s}^{n}} d \widetilde{K_{s}^{n}} \mid \mathcal{F}_{t}\right] \leq 0
$$

Furthermore, we have

$$
\left(\widetilde{y_{0}^{n}}\right)^{2}+K_{1} \mathcal{E}^{g}\left(\int_{0}^{T} e^{(s-t)} \beta{\widetilde{y_{t}^{n}}}^{2} d s\right)+K_{2} \mathcal{E}^{g}\left(\int_{0}^{T} e^{(s-t)}{\widetilde{z_{s}^{n}}}^{2} d s\right) \leq e^{\beta(T-t)} \mathcal{E}^{g}\left(\varepsilon_{n}^{2}\right) .
$$

For $\left|\xi_{i}\right| \leq|\eta|, i=1,2, \cdots, \eta \in L^{2}\left(\mathcal{F}_{T}\right)$, by the Lebesgue dominated convergence theorem, we have

$$
\lim _{n \rightarrow \infty} \mathcal{E}^{g}\left(\varepsilon_{n}^{2}\right)=\lim _{n \rightarrow \infty} \mathcal{E}^{g}\left(\frac{s_{n}}{n}-C\right)^{2}=0 .
$$

Therefore,

$$
\lim _{n \rightarrow \infty} \widetilde{y_{0}^{n}}=0
$$

i.e.,

$$
\lim _{n \rightarrow \infty} \mathcal{E}^{g}\left(\frac{s_{n}}{n}\right)=\mathcal{E}^{g}(C)
$$

\section{ACKNOWLEDGEMENTS}

The authors thank Professor Shaozhu Chen for his valuable suggestions. The authors also thank the anonymous referee for insightful comments and advice that led to an improved version of this paper.

\section{REFERENCES}

1. Z. Chen, R. Kulperger and L. Jiang, Jensens' inequality for g-expectation, Part I. C. R. Math. Acad. Sci. Paris Sér. I Math. 337 (11) (2003) 725-730. MR2030410 (2004k:60162)

2. Z. Chen, R. Kulperger and L. Jiang, Jensens' inequality for g-expectation, Part II. C. R. Math. Acad. Sci. Paris Sér. I Math. 337 (12) (2003) 797-800. MR2033122 (2004k:60163)

3. J. Cvitanić and I. Karatzas, Backward stochastic differential equations with reflection and Dynkin games, The Annals of Probability 24 (4) (1996), 2024-2056. MR.1415239 (97h:93080)

4. N. El Karoui, C. Kapoudjian, E. Pardoux, S. Peng and M.C. Quenez, Reflected solutions of backward SDE's and related obstacle problems for PDE's, The Annals of Probability 25 (1997) 702-737. MR1434123 (98k:60096)

5. N. El Karoui, S. Peng and M.C. Quenez, Backward stochastic differential equations in finance, Math. Finance 7 (1997) 1-71. MR1434407 (98d:90030)

6. L. Jiang and $\mathrm{Z}$. Chen, A result on the probability measures dominated by g-expectation, Acta Mathematicae Applicatae Sinica, English Series 20 (3) (2004) 507-512. MR2086771 (2006a:60106)

7. L. Jiang, and Z. Chen, On Jensens' inequality for g-expectation, Chinese Ann. Math. Ser. 25B, 3 (2004) 401-412. MR2086132 (2005h:60168)

8. E. Pardoux and S. Peng, Adapted solution of a backward stochastic differential equation, Systems \& Control Lett. 14 (1990) 55-61. MR1037747 (91e:60171) 
9. S. Peng, Backward SDE and related g-expectation, in Backward Stochastic Differential Equations, El Karoui, N. and Mazliak, L., eds., Pitman Research Notes in Mathematics Series, 364: 141-159, Longman, Harlow, 1997. MR.1752671 (2000k:60003)

10. A.N. Shiryayev, Probability, Graduate Texts in Math., vol. 95, Springer, Berlin-HeidelbergNew York, 1984. MR0737192(85a:60007)

School of Mathematics and Statistics, Shandong University at Weihai, Weihai 264209, People's Republic of China

E-mail address: bingyang@sdu.edu.cn

School of Mathematics and Statistics, Shandong University at Weihai, Weihai 264209, People's Republic of China

E-mail address: xiao_hua@sdu.edu.cn 